\title{
INFLUENCIA DE LA INTENSIDAD DE RALEOS EN LA PERFORMANCE DE UN HARVESTER DE RUEDAS EN EL NORESTE ARGENTINO
}

\author{
Patricio Mac Donagh ${ }^{1}$, Eduardo Hildt ${ }^{2}$, Ramón A. Friedl ${ }^{3}$, Constantino Zaderenko ${ }^{4}$, \\ Diego A. Alegranza \\ ${ }^{1}$ Ing. Forestal, M.Sc., Facultad de Ciencias Forestales, UNaM, Eldorado, Misiones, Argentina - patricio.macdonagh@gmail.com \\ ${ }^{2}$ Est. Ing. Forestal, Facultad de Ciencias Forestales, UNaM, Eldorado, Misiones, Argentina - eduardohildt@ gmail.com \\ ${ }^{3}$ Ing. Forestal, M.Sc., Facultad de Ciencias Forestales, UNaM, Eldorado, Misiones, Argentina - afriedl@ facfor.unam.edu,ar \\ ${ }^{4}$ Ing. Aeronáutico, M.T., Facultad de Ciencias Forestales, UNaM, Eldorado, Misiones, Argentina - zaderenko@ facfor,unam,edu,ar \\ ${ }^{5}$ Ing. Forestal, Actitud Forestal Estudio de Ingeniería y Representaciones, Eldorado, Misiones, Argentina - actitudforestal@ gmail.com
} Recebido para publicação: 28/12/2012 - Aceito para publicação: 30/09/2013

\begin{abstract}
Resumen
Se analizóla performancede un "harvester" sobre ruedas en plantaciones de Pinus spp. en la provincia de Misiones, en tres operaciones: primer raleo en 4 líneas a la vez (R1-4L), primer raleo en 7 líneas a la vez (R1-7L), y segundo raleo (R2).Las operaciones se realizaron en terrenos suavemente ondulados. Se analizaron tiempos y rendimientos del harvester realizando, volteo, desrame y trozado. El harvester presentó una productividad media de $14,2 \mathrm{~m}^{3} / \mathrm{h}$ ef para la situación $\mathrm{R} 1-4 \mathrm{~L}, 12,15 \mathrm{~m}^{3} / \mathrm{h}$ ef para la situación R1-7L y $30,81 \mathrm{~m}^{3} / \mathrm{h}$ ef en la situación R2. Se ajustaron modelos matemáticos empleando como principales variables el DAP y el volumen por árbol. Se obtuvieron coeficientes de determinación ajustados $\left(\mathrm{R}^{2}\right.$ aj) del orden del $65 \%$ para $\mathrm{R} 1-4 \mathrm{~L}, 59 \%$ para $\mathrm{R} 1-7 \mathrm{~L}$ y del $41 \%$ para $\mathrm{R} 2$. El costo de producción total presentó un comportamiento inverso al de la productividad. El tipo de intervención y el volumen de los árboles cosechados fueron los principales factores que condicionaron la productividad de la operación de cosecha. La operación R2 presentó el menor costo de las tres, mientras que la operación R1-4L presentó un costo inferior al de la alternativa evaluada (R1-7L).

Palabras claves: Pinus; cosecha forestal; aclareos.
\end{abstract}

\section{Resumo}

Influência da intensidade do desbaste no desempenho de um harvester sobre pneus no nordeste Argentino. Foi analisado o desempenho de um "harvester" sobre pneus em plantios de Pinus spp. na província de Misiones, em três operações: primeiro desbaste em quatro linhas (R1-4L), primeiro desbaste em sete linhas (R1-7L) e segundo desbaste (R2). As operações foram realizadas em relevos suaves. Foram analisados tempos e rendimentos do harvester realizando corte, desgalhamento e traçamento. O harvester apresentou uma produtividade media de $14,2 \mathrm{~m}^{3} / \mathrm{h}$ ef para a situação R1-4L, $12,15 \mathrm{~m}^{3} / \mathrm{h}$ ef para R1-7L e $30,81 \mathrm{~m}^{3} / \mathrm{h}$ ef em R2. Foram ajustados modelos matemáticos empregando como principais variáveis o DAP e o volume por árvore. Foram obtidos coeficientes de determinação ajustados $\left(\mathrm{R}^{2} \mathrm{aj}\right)$ da ordem de $65 \%$ para $\mathrm{R} 1-4 \mathrm{~L}, 59 \%$ para $\mathrm{R} 1-7 \mathrm{~L}$ e de $41 \%$ para $\mathrm{R} 2$. O custo de produção total apresentou um comportamento inverso ao da produtividade. $\mathrm{O}$ tipo de operações e $\mathrm{o}$ volume das árvores colhidas foram os principais fatores que afetaram a produtividade das operações de colheita. A operação R2 apresentou o menor custo das três, enquanto que a operação R1-4L apresentou um custo inferior ao da alternativa avaliada (R1-7L).

Palavras-chave: Pinus; colheita florestal; desbastes.

\section{INTRODUCCIÓN}

En la cosecha forestal de bosques implantados en el Nordeste Argentino (NE) la mecanización de las actividades ya ha alcanzado a todas las etapas del ciclo productivo y sigue una tendencia creciente. Esto se debe en parte a que aquela tendencia es la de cosechar árboles de bajo volumen individual, donde los escasos márgenes de ganancia exigen un proceso constante de mejora de los rendimientos y reducción de los costos de producción. 
En las operaciones de raleo es donde la mecanización presenta más interrogantes, sobre todo cuando se relaciona la intensidad de raleo a la performance de la operación. En estas situaciones, se plantea desde la viabilidad económica de realizar la operación, hasta alguna combinación entre raleos sistemáticos y selectivos, que permita mejorar el resultado.

El harvester de ruedas ha sido incorporado a las operaciones de raleos como maquinaria de corte para condiciones que exigen precisión en la obtención de productos, alta productividad y pocos daños a la masa remanente (MCNEEL; RUTHERFORD, 1994). Los principales factores que afectan a la productividad de un harvester son el volumen de los árboles, el número de productos a obtener y las distancias que debe recorrer en la forestación (SILVA et al., 2010; MARTINS et al., 2009).

Parra y Carey (2000) indican que aunque estos equipos son tolerantes a terrenos con relieve escarpado, su potencial productivo disminuye sustancialmente cuando la pendiente supera el $30 \%$.

Mac Donagh et al. (2013), analizando harvesters sobre orugas realizando tala rasa en forestaciones con y sin raleos previos no encontraron diferencias significativas en el costo de producción para ambas situaciones. Sin embargo, Martins et al. (2009) indican que el mayor espaciamiento existente entre los árboles en un rodal que presenta raleos previos afecta positivamente a la productividad de la operación debido al mayor volumen individual de los árboles.

Para operaciones de corte raso, es más frecuente encontrar modelos de productividad, tales como los que presentan Bramucci y Seixas (2002).En ese caso ajustaron modelos para harvesters realizando cosecha de Eucalyptus spp. en Brasil, determinando que el volumen por árbol es la variable que mejor se correlaciona con la productividad, con coeficientes de determinación superiores a 0,75.

Simões et al. (2010), evaluando un harvester Caterpillar 320 CL con cabezal Valmet 965-BR determinaron que el Diámetro a la Altura del Pecho (DAP) determina cerca del 50\% de la productividad de la máquina realizando talas rasas en Eucalyptus spp de 72 meses de edad.

Para el caso de harvesters de ruedas, Martins et al. (2009), evaluaron la influencia del volumen por árbol y el espaciamiento sobre el rendimiento de un John Deere 1270 con cabezal Waratah 762. Estos autores hallaron que el volumen medio por árbol, el espaciamiento y las características del harvester son los factores que mejor explican el rendimiento de esta máquina realizando tala rasa en plantaciones de Eucalyptus spp, obteniendo modelos lineales con coeficientes de determinación del $98 \%$ utilizando el volumen de árbol medio como variable regresora.

Nurminen et al. (2006), han analizado rendimientos de distintos harvester de ruedas realizando talas rasas y raleos en bosques mixtos de Finlandia. Encontraron que el volumen medio del árbol es la principal variable que afecta a la productividad, siendo esta satisfactoriamente explicada mediante modelos cuadráticos.

Sirén y Aaltio (2003) determinaron que el volumen de madera extraída en un raleo y la cantidad de productos elaborados son variables explicativas de la productividad de harvesters realizando aclareos en bosques nativos de Finlandia. Eliasson (2000) encontró que en bosques de Picea abies de Suecia, el tiempo medio de cosecha aumenta al incrementarse el volumen medio de los árboles y al aumentar el espaciamiento entre estos. Sin embargo, estas operaciones son diferentes a las encontradas en Argentina, donde la realización de raleos en plantaciones forestales implica una combinación de cortas sistemáticas abriendo vías de acceso y cortas selectivas en la masa remanente.

El objetivo de este estudio fue desarrollar modelos de productividad y costos para un harvester sobre ruedas trabajando con dos intensidades de raleo diferentes en plantaciones de Pinus taeda en el NE Argentino.

\section{MATERIALES Y MÉTODOS}

Se realizó un estudio de caso sobre un harvestercon ruedas trabajando en la realización de raleos en plantaciones forestales de Pinus taeda analizando operaciones en primeros raleos y en segundos raleos.

El trabajo fue desarrollado en la zona norte de la provincia de Misiones, en plantaciones de cercanas a la localidad Eldorado ( $26^{\circ} 30^{\prime}$ Sur; $54^{\circ} 43^{\prime}$ Oeste). Los terrenos son suavemente ondulados con pendientes entre el 0 y $5 \%$. El clima subtropical húmedo es definido como "Caf" según la clasificación climática de Köppen, con una temperatura media anual de $20{ }^{\circ} \mathrm{C}$, una amplitud de $11{ }^{\circ} \mathrm{C}$, una precipitación de $2000 \mathrm{~mm}$ por año y sin estación seca. Los suelos pertenecen al gran grupo de los Kandiudultes, conocidos como "tierras coloradas" (Instituto Nacional de Tecnología Agropecuaria, 
1990), habiendo sectores que por su posición topográfica presentan suelos con características hidromórficas.

El harvester analizado, marca John Deere, modelo 1070 D, provisto con un cabezal cosechador modelo H 754, consiste en una maquina base articulada con tracción 6x6, motor John Deere 6068HTJ con una potencia nominal de $136 \mathrm{~kW}$, sistema rodante de neumáticos 600 × 22.5, 16 delanteros y 750 x 26.5 , 20 traseros; grúa telescópica con un alcance de total de $10 \mathrm{~m}$ y un cabezal de $820 \mathrm{Kg}$ de peso con un diámetro de corte máximo de $55 \mathrm{~cm}$. El equipo posee un peso total de $14100 \mathrm{Kg}$.

Las situaciones de trabajo estudiadas fueron las siguientes:

- Primer Raleo interviniendo 4 líneas a la vez (R1-4L): La operación de raleo fue realizada por un mismo operario en una forestación de 5 años de edad, $15,2 \mathrm{~cm}$ de DAP medio, una altura media de $8,9 \mathrm{~m}$ y un volumen de árbol medio de $0,079 \mathrm{~m}^{3} \mathrm{cc}$.En este caso la máquina avanzó realizando el raleo sistemático en una línea y raleo selectivo en los árboles marcados en las dos líneas adyacentes a cada lado de la vía de acceso abierta (Figura 1 a).

- Primer Raleo interviniendo 7 líneas a la vez (R1-7L): Esta operación de raleo fue realizada en una forestación de cuatro años de edad, con un DAP medio de 15,6 cm, una altura media de 7,9 $\mathrm{m}$ y un volumen de árbol medio de $0,076 \mathrm{~m}^{3} \mathrm{cc}$. En este caso la máquina avanzó abriendo realizando el raleo sistemático en un lineo y raleo selectivo en los árboles marcados en 3 y 4 líneos adyacentes a cada lado de la vía de acceso abierta. Para ello la máquina ingresaba parcialmente en las líneas adyacentes hasta alcanzar con el cabezal a los árboles más lejanos (Figura 1,b).

- Segundo Raleo (R2): En esta situación se realizó un segundo raleo selectivo en una plantación de diez años de edad, con un DAP medio de $24,8 \mathrm{~cm}$ y $15,7 \mathrm{~m}$ de altura media y un volumen de árbol medio de $0,354 \mathrm{~m}^{3} \mathrm{cc}$. La máquina operó ingresando por una vía de acceso y realizando el raleo selectivo de 4 líneas al mismo tiempo.

$\mathrm{R} 1-4 \mathrm{~L}$

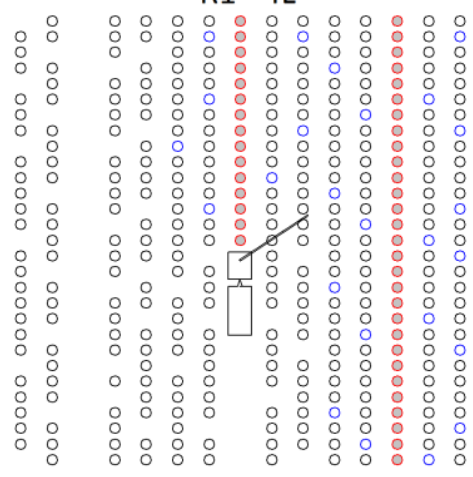

a
R1 - 7L
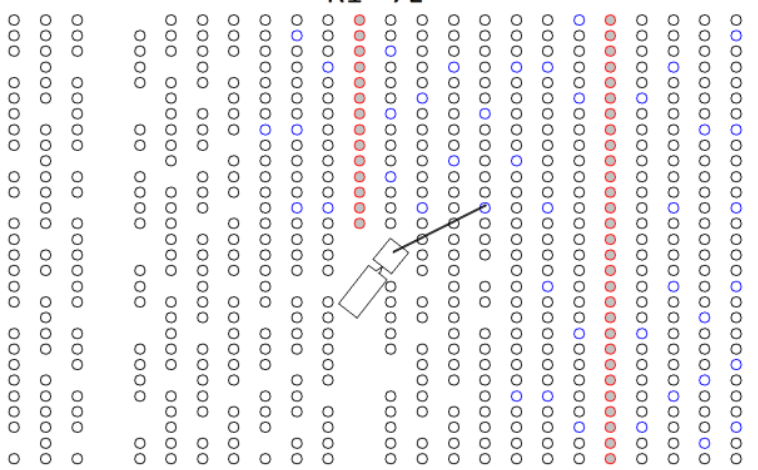

b

Figura 1. Diagrama de la técnica de trabajo utilizada para las operaciones de Primer Raleo en 4 y 7 líneas.

Figura 1. Diagrama da técnica utilizada para a primeira operação de desbaste em quatro e sete linhas.

El sistema de cosecha utilizado por la empresa fue el de toras cortas (Cut-to-length). Se produjeron trozas de largo variable y $8 \mathrm{~cm}$ en punta fina con destino al triturado para la obtención de celulosa, y trozas para aserrado de 2,7 a 4 metros de largo con $16 \mathrm{~cm}$ en punta fina.

En todos los casos, con anterioridad a la realización de los raleos se midió el DAP de la totalidad de los árboles y se los individualizó mediante una matriz de líneas y posiciones para permitir su posterior identificación durante el aprovechamiento y la medición de tiempos. La altura de los árboles fue estimada mediante funciones hipsométricas ajustadas para cada sitio en particular. El volumen con corteza de cada árbol fue ajustado mediante la función de volumen para Pinus taeda publicada por Crechi et al. (2006).

Los árboles cosechados presentaron un volumen medio de 0,093 $\mathrm{m}^{3}$ (Desvío Estándar (SD): 0,06 $\left.\mathrm{m}^{3}\right)$ con corteza para la situación R1 - 7L, 0,094 $\mathrm{m}^{3} \mathrm{cc}\left(\mathrm{SD}: 0,06 \mathrm{~m}^{3}\right.$ ) para la situación R1 - 7L y 0,307 
$\mathrm{m}^{3} \mathrm{cc}\left(\mathrm{SD}: 0,12 \mathrm{~m}^{3}\right.$ ) para la situación R2. La situación R2 presentó diferencias respecto a las situaciones de primer raleo, significativas al 5\% de probabilidad según el test de Tukey.

Los estudios de tiempos se realizaron utilizando un cronómetro y según la metodología de toma de tiempos individuales, discriminando entre actividades productivas efectivas y generales. La unidad experimental fue el ciclo operacional, comprendiendo la localización y movimiento hasta el árbol, su volteo, desrame y trozado formado pilas con los productos. El número mínimo de observaciones necesarias, fue calculado mediante las siguiente expresión (1), de modo de proporcionar un error de muestreo máximo del 5\%.

El error absoluto de muestreo:

$$
E=t * s / \sqrt{n}
$$

El error relativo de:

$$
\mathrm{E} \%=\mathrm{E} / \mathrm{x} * 100
$$

Dónde: t: Valor de la distribución t Student;

s: Desvío estándar de la muestra.;

n: Tamaño de la muestra; x: Promedio de la muestra.

Para cada situación de trabajo se calculó la productividad y la eficiencia operacional del equipo. Mediante análisis de regresión lineal múltiple y no lineal se ajustaron modelos predictivos de la productividad por hora efectiva, obtenidos de trabajos anteriores (ERBETTA et al., 2006) o generados in situ mediante combinación de variables, utilizando como principales variables el DAP y el volumen individual de los árboles cosechados.

La productividad del harvester fue determinada en metros cúbicos con corteza por hora de trabajo efectiva, conforme con la siguiente expresión (2):

$$
P E F=V T / T A E
$$

Dónde: PEF = Productividad del harvester por hora de trabajo efectivo $\left(\mathrm{m}^{3} \mathrm{cc} / \mathrm{h}\right.$ ef $)$;

$\mathrm{VT}=$ Volumen total cosechado $\left(\mathrm{m}^{3}\right)$;

$\mathrm{TAE}=$ Tiempo total efectivo de trabajo, en horas efectivas.

Las diferentes situaciones fueron probadas mediante el análisis de la varianza y la aplicación del Test de Tukey al 5\% de probabilidad.

El costo horario de operación fue determinado por medio de la metodología propuesta por la Organización de las Naciones Unidas para la Alimentación y la Agricultura(FAO) (MALINOVSKI, 1983; STÖHR, 1977), en base a la información provista por la empresa propietaria del equipo referida al valor de adquisición, antigüedad, vida útil estimada, importe típico de insumos y reparaciones, consumo de combustible, aceites y cadenas, salario, aportes sociales y elementos de protección personal de los operarios.

En base al costo horario de operación se calcularon los costos unitarios medios de producción efectivos y totales para cada equipo según las expresiones (3) y (4) respectivamente.

$$
\mathrm{CPE}=\mathrm{COH} / \mathrm{PEF}
$$

Dónde: $\mathrm{CPE}=$ Costo de producción efectivo (US\$/Tn);

$\mathrm{COH}=$ Costo horario de operación $(\mathrm{US} \$ \mathrm{~h})$;

$\mathrm{PEF}=$ Productividad del harvester por hora de trabajo efectivo $\left(\mathrm{m}^{3} \mathrm{cc} / \mathrm{h}\right.$ ef $)$.

$$
C P T=C O H /(P E F * E F)
$$

Dónde: $\mathrm{CPT}=$ Costo de producción total (US\$/Tn);

$\mathrm{COH}=$ Costo horario de operación $(\mathrm{US} \$ / \mathrm{h})$;

$\mathrm{PEF}=$ Productividad del harvester por hora de trabajo efectivo $\left(\mathrm{m}^{3} \mathrm{cc} / \mathrm{h}\right.$ ef $)$;

$\mathrm{EF}=$ Eficiencia operacional. 


\section{RESULTADOS Y DISCUSIÓN}

Se relevaron un total de 1562 ciclos en la situación R1 - 4L, 1131 ciclos enR1 - 7L, y 327 ciclos para la situación de R2.

En la tabla 1 se presentan los resultados de productividad por hora de trabajo efectivo y eficiencia para las tres situaciones de trabajo. Se observaron diferencias estadísticamente significativas entre la productividad efectiva de los primeros raleos con 4 y 7 líneas. La velocidad de procesamiento de los árboles fue de 151 arb/h.ef. para la situación R1 - 4L y de 128 arb/h.ef. para R1 - 7L. En la situación R2 la velocidad de procesamiento fue de $99 \mathrm{arb} / \mathrm{h}$.ef.

Tabla 1. Resultados de productividad y eficiencia.

Tabela 1. Resultados de produtividade e eficiência.

\begin{tabular}{lcc}
\hline Operación & $\begin{array}{c}\text { Productividad } \\
\left(\mathbf{m}^{\mathbf{3}} / \mathbf{h} \text { ef }\right)\end{array}$ & $\begin{array}{c}\text { Eficiencia } \\
\text { Operacional }(\%)\end{array}$ \\
\hline R1 4 líneas & $14,20 \mathrm{~b}$ & 0,65 \\
R1 7 líneas & $12,15 \mathrm{c}$ & 0,74 \\
R2 & $30,81 \mathrm{a}$ & 0,32 \\
\hline Las medias seguidas con diferentes letras difieren estadísticamente entre sí, por el Test de \\
Tukey al 5\% de probabilidad.
\end{tabular}

Estos resultados son coincidentes con lo establecido por Siren y Aaltio (2003), ya que la mayor productividad efectiva observada en la situación R1 - 4L está relacionada a una mayor intensidad de raleo, con una mayor frecuencia de líneas de raleo sistemático y menores distancias de desplazamiento para la realización de la corta selectiva.

La mayor productividad observada en la situación en que se ralean 4 líneas a la vez puede deberse a que existieron diferencias significativas entre el tiempo medio por ciclo que insume la utilización de la grúa al límite máximo de su rango operativo al trabajar raleando 7 líneas a la vez $(0,467$ min) respecto de la situación en que se ralean 4 líneas a la vez (0,397 min). La extensión telescópica de la grúa insume un mayor tiempo al ciclo de corta y reduce la capacidad del cabezal para controlar el volteo y el movimiento de los árboles.

Respecto a la baja eficiencia operacional observada en R2, esta se debe en parte a una mayor incidencia de desperfectos técnicos. Entre las dos situaciones de primer raleo apenas hubo diferencias de eficiencia operacional, debidas principalmente a un menor tiempo de retornos carreteando al cosechar 7 líneas a la vez.

Con los resultados obtenidos de los estudios de tiempos y rendimientos se ajustaron modelos predictivos de la productividad por hora de trabajo efectiva para las tres situaciones analizadas. Mediante análisis de regresión se probaron 20 modelos obtenidos de la literatura o generados mediante combinación de variables. El DAP y el volumen del árbol fueron las variables que mejor explicaron el incremento en la productividad por hora efectiva al aumentar el tamaño de los árboles, coincidiendo con las experiencias de Bramucci y Seixas (2002), Simões et al. (2010) trabajando con harvester sobre orugas y Martins et al. (2009), Nurminen et al. (2006) trabajando con harvesters sobre ruedas.

Los modelos fueron elegidos buscando un alto coeficiente de determinación, bajo error estándar de estimación, bajo valor del indicador PRESS y adecuada distribución de los residuos.

Para la situación R1 -4Lse obtuvo el mejor ajuste con un modelo lineal múltiple donde el DAP y el logaritmo del DAP fueron las variables regresoras. En la situación R1 - 7L el mejor modelo fue ajustado mediante una expresión potencial, con el volumen individual del árbol como variable regresora. En la situación R2 el mejor ajuste se obtuvo con un modelo cuadrático donde el volumen individual del árbol fue la variable regresora. Estos resultados se presentan en la tabla 2. En todos los modelos elegidos los coeficientes ajustados resultaron significativos al 5\% de probabilidad.

En las figuras 2 y 3 se representan los mejores modelos ajustados para cada situación.

En las situaciones de primer raleo se observa un incremento fuerte de la productividad al aumentar el diámetro y volumen individual de los árboles, que decrece hasta conformar una relación prácticamente lineal. Esto se debe a que los incrementos en el diámetro delos árboles se traducen en aumentos cuadráticos del volumen de los fustes cosechados en cada ciclo. A volúmenes mayores a $0,2 \mathrm{~m}^{3}$ 
el peso de los árboles comienza a limitar la velocidad de trabajo, estabilizándose la curva de producción en una tendencia lineal. No se observaron limitaciones técnicas debido al peso de los árboles que reduzcan la productividad en el rango de volúmenes evaluado. Estas funciones poseen un comportamiento acorde a las obtenidas por Nurminen et al. (2006) en cortas totales y raleos de bosques naturales en Finlandia quienes trabajaron con un volumen por árbol similar pero una menor productividad debido a las condiciones de mayor espaciamiento, pendientes y obstáculos existentes en el bosque natural.

Tabla 2. Modelos ajustados e indicadores estadísticos.

Tabela 2. Modelos ajustados e indicadores estatísticos.

\begin{tabular}{|c|c|c|c|c|c|c|c|}
\hline \multirow{2}{*}{ Operación } & \multirow{2}{*}{ Modelo } & \multicolumn{3}{|c|}{ Coeficientes } & \multicolumn{3}{|c|}{ Indicadores estadísticos } \\
\hline & & b0 & b1 & b2 & $\mathbf{R}^{2}$ aj & Syx & PRESS \\
\hline $\mathrm{R} 1-4 \mathrm{~L}$ & $\mathrm{VOL} / \mathrm{h}=\mathrm{b} 0+\mathrm{b} 1 * \mathrm{DAP}+\mathrm{b} 2 * \ln (\mathrm{DAP})$ & 3,6698 & 1,9875 & $-7,3029$ & 0,65 & 4,61 & 33214,98 \\
\hline $\mathrm{R} 1-7 \mathrm{~L}$ & $\mathrm{VOL} / \mathrm{h}=\mathrm{b} 0 *(\mathrm{Vol} / \mathrm{árb})^{\wedge} \mathrm{b} 1$ & 72,2142 & 0,7168 & & 0,59 & 4,55 & \\
\hline $\mathrm{R} 2$ & $\mathrm{VOL} / \mathrm{h}=\mathrm{b} 0+\mathrm{b} 1 *(\mathrm{Vol} / \mathrm{ar} \mathrm{b})^{\wedge} 2$ & 26,1118 & 74,7508 & & 0,41 & 9,63 & 30634,06 \\
\hline
\end{tabular}

Dónde: VOL/h: Productividad efectiva (m³/h.ef.); DAP: Diámetro a la altura del pecho $(\mathrm{cm}) ;$ Vol/árb: Volumen individual de los árboles $\left(\mathrm{m}^{3}\right)$.

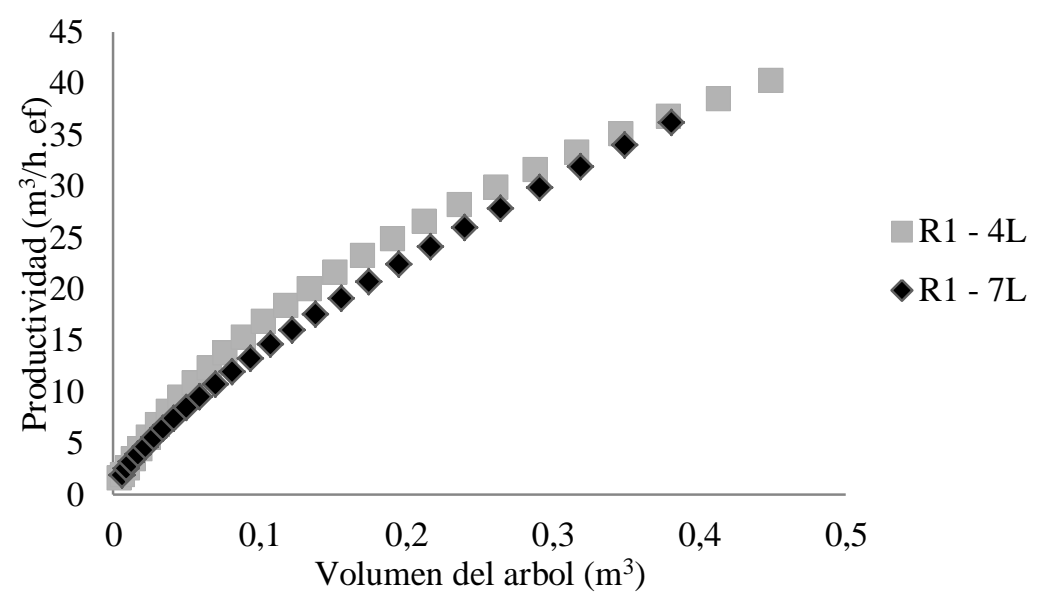

Figura 2. Comportamiento delos modelos seleccionado para la operación de primer raleo en 4 y 7 líneas.

Figura 2. Comportamento dos modelos selecionados para a primeira operação de desbaste em quatro e sete linhas.

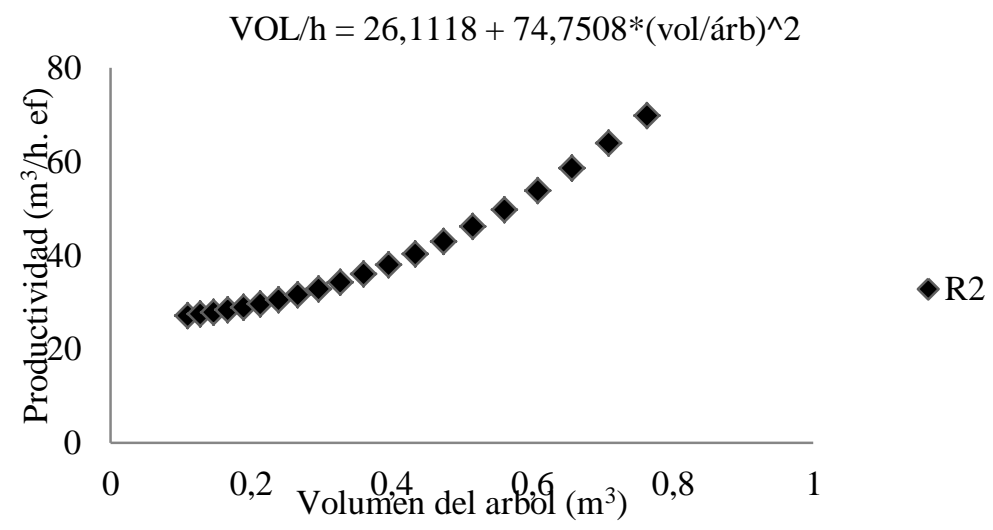

Figura 3. Comportamiento del modelo seleccionado para la operación de segundo raleo.

Figura 3. Comportamento do modelo selecionado para segundo desbaste. 
Estos resultados coinciden también con los obtenidos por Martins et al. (2009), quien trabajando con una máquina mayor, aunque de diseño similar obtuvo relaciones lineales con productividades de 20 $\mathrm{m}^{3} / \mathrm{h}$. ef.para árboles de $0,2 \mathrm{~m}^{3}$.

En la situación R2 la productividad muestra una correlación positiva con el aumento del tamaño de los árboles, sin apreciarse limitaciones en la capacidad productiva del harvester. Esto demuestra que con esta máquina es posible la realización de raleos en forestaciones con volúmenes de árbol individuales cercanos a $0,8 \mathrm{~m}^{3}$ trabajando con un alcance de la grúa de alrededor de $5 \mathrm{~m}$.

Del cálculo de los costos resultó que la operación más costosa fue el primer raleo, especialmente cuando la maquina operaba procesando 7 líneas a la vez. La operación de segundo raleo posee un menor costo por tonelada de madera elaborada debido a la mayor productividad lograda al elaborar árboles con un mayor volumen individual en condiciones de espaciamiento más favorables a la operación del harvester. Aun considerando su baja eficiencia operacional esta diferencia sigue existiendo, como puede observarse en la tabla 3.

Tabla 3. Costo operacional y de producción para el harvester.

Tabela 3. Produção e custos operacionais para o harvester.

\begin{tabular}{lccc}
\hline Operación & $\begin{array}{c}\text { Costo operacional } \\
\text { (US\$/h) }\end{array}$ & $\begin{array}{c}\text { Costo (US\$/Tn) } \\
\text { (Prod. Efectiva) }\end{array}$ & $\begin{array}{c}\text { Costo (US\$/Tn) } \\
\text { (Prod. General) }\end{array}$ \\
\hline R1 - 4 Líneas & 47,05 & $3,31 \mathrm{~b}$ & $5,10 \mathrm{~b}$ \\
R1 - 7 Líneas & 47,05 & $3,87 \mathrm{c}$ & $5,22 \mathrm{~b}$ \\
R2 & 47,05 & $1,53 \mathrm{a}$ & $4,72 \mathrm{a}$ \\
\hline Las medias seguidas con diferentes letras difieren estadísticamente entre sí, por el Test de Tukey \\
al 5\% de probabilidad.
\end{tabular}

En este contexto, considerando la productividad obtenida por los modelos seleccionados y el costo medio de producción para cada operación, en las situaciones de primer raleo se observa una reducción exponencial del costo de producción con el aumento del tamaño de los árboles y por lo tanto de la productividad efectiva, hasta alcanzar un límite en que el costo se estabiliza. Esto puede observarse en las figuras 4 y5, donde se presentan las curvas de producción y costos para cada situación.

En la situación de segundo raleo y para los tamaños de árboles estudiados, el costo de producción sufre una reducción paulatina y constante al aumentar el tamaño de los árboles procesados.

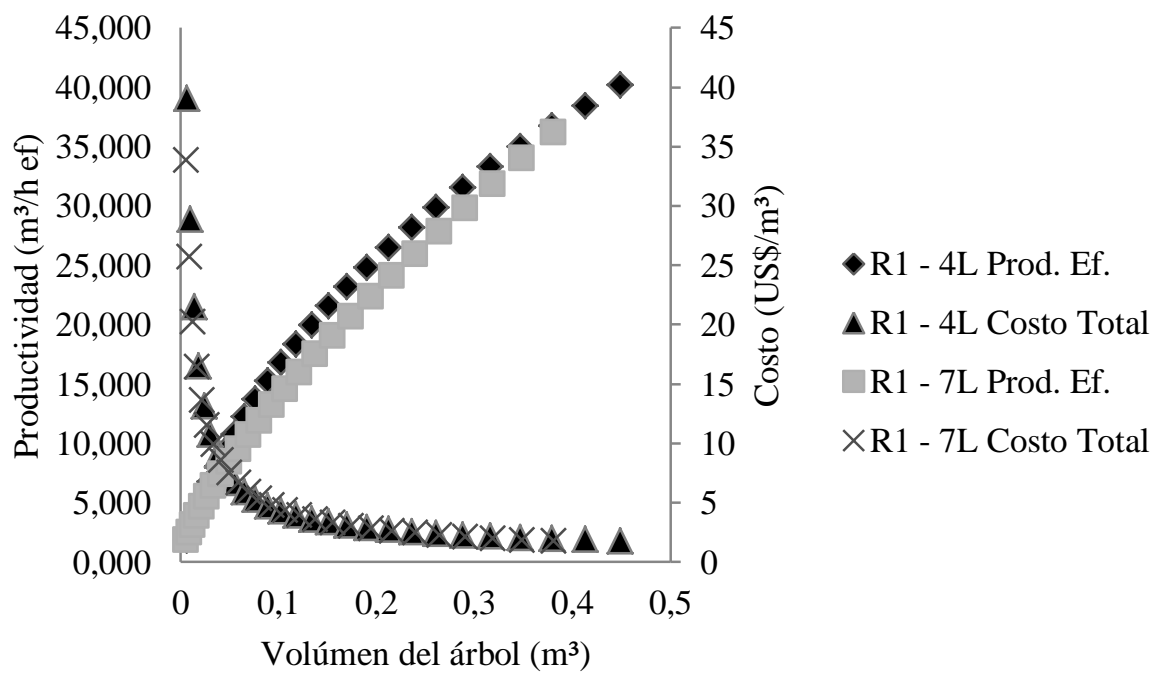

Figura 4. Productividad y costo del harvester en función del volumen de los árboles para la operación de primer raleo en 4 y 7 líneas.

Figura 4. Produtividade e custos do harvester com base no volume de árvores para a operação de primeiro desbaste em quatro e sete linhas. 


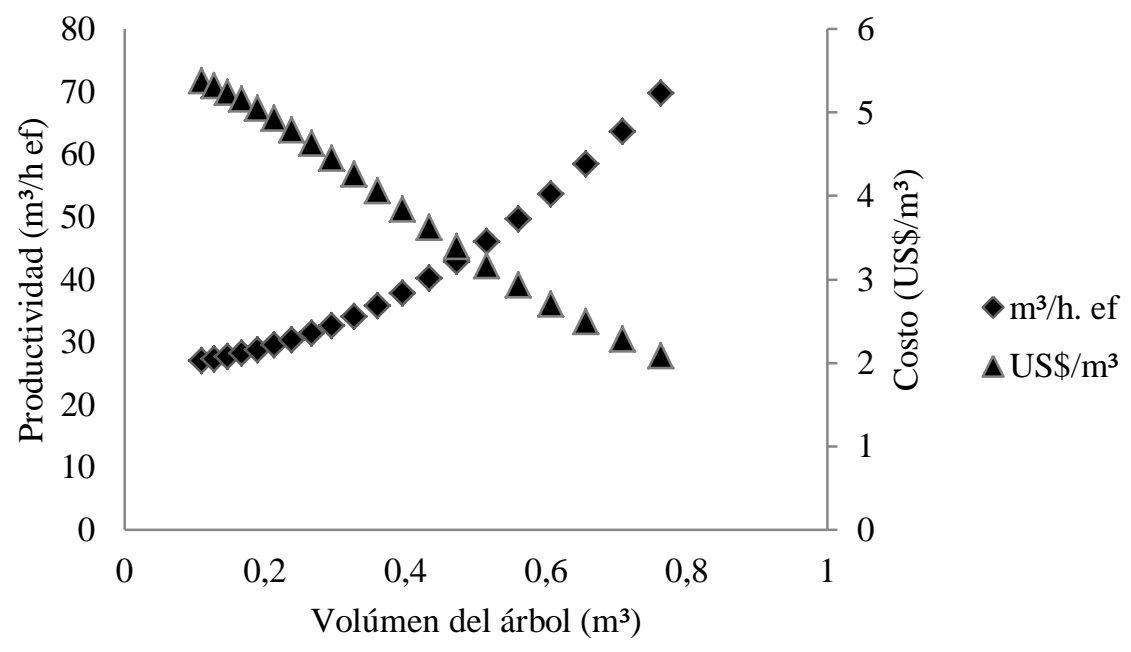

Figura 5. Productividad y costo del harvester en función del volumen de los árboles para la operación de segundo raleo.

Figura 5. Produtividade e custos do harvester com base no volume de árvores para a operação de segundo desbaste.

\section{CONCLUSIONES}

- El harvester sobre ruedas analizado presentó un mejor comportamiento en los primeros raleos en que se intervienen 4 líneas a la vez. En la situación en que se intervienen 7 líneas la máquina no alcanza las líneas más externas y debe realizar maniobras que reducen su productividad.

- No se aprecia una reducción de la productividad debido a limitaciones técnicas importantes relacionadas el aumento del peso de los árboles, dentro del rango de volúmenes observados.

- El volumen de los árboles es el factor más importante a considerar durante la planificación de las operaciones de raleo con harvester, debido a que es el principal condicionante de la productividad.

- La productividad efectiva del equipo aumenta al trabajar con una mayor intensidad raleo mediante el corte sistemático de líneos.

- El diámetro a la altura del pecho y el volumen individual de los árboles fueron las variables que mejor estimaron la productividad efectiva del equipo. Los bajos valores observados en el coeficiente de determinación indican que existen otras fuentes de variación que deberían ser analizadas en estudios posteriores.

\section{REFERENCIAS}

BRAMUCCI, M.; SEIXAS, F. Determinação e quantificação de fatores de influência sobre a produtividade de "harvesters" na colheita florestal. Scientia Forestalis, Piracicaba, v. 30, n. 62, p. 62 74, 2002.

CRECHI, E.; FASSOLA, H.; KELLER, A.; BARTH, S. Modelos de estimación del volumen individual de árboles con y sin corteza de Pinus taeda L. para la zona de Norte de Misiones, Argentina. In: JORNADAS TÉCNICAS FORESTALES Y AMBIENTALES, 12., 2006, Eldorado. Anales... Eldorado, 2006. p. 14.

ELIASSON, L. Effects of establishment and thinning of shelterwoods on harvester performance. International Journal of Forest Engineering, Seattle, v. 11, n. 1, p. 21 - 27, 2000.

ERBETTA, D.; COSTA, R.; GONZÁLEZ, J.; MAC DONAGH, P.; DURAN, D. Modelos predictivos en la productividad de Feller Buncher Bell Ultra C. In: CONGRESO LATINOAMERICANO IUFRO, 2., 2006, La Serena, Chile Anales... La Serena, 2006. p. 6. 
PEÑA ZUBIATE, C. A.; PINEDO, D. M.; Atlas de suelos de la República Argentina. Buenos Aires: Secretaría de Agricultura, Ganadería y Pesca (SAGyP) - Instituto Nacional de Tecnología Agropecuaria (INTA), 1990. v. 2, 600 p.

MALINOVSKI, J. Metodologia do custo-hora para máquinas florestais. In: DIETZ, P. IV Curso de Atualização sobre Sistemas de Exploração e Transporte Florestal. Curitiba, 1983. p. 57 - 70.

MARTINS, R. J.; SEIXAS, F.; STAPE, J. L. Avaliação técnica e econômica de um harvester trabalhando em diferentes condições de espaçamento e arranjo de plantio em povoamento de eucalipto. Scientia Forestalis, Piracicaba, v. 37, n. 83, p. 253 - 263, 2009.

MCNEEL, J. F.; RUTHERFORD, D. Modelling Harvester-Forwarder System Performance in a Selection Harvest. International Journal of Forest Engineering, Seattle, v. 6, ed. 1, 1994.

NURMINEN, T.; KORPUNEN, H.; JORI, U. Time Consumption Analysis of the Mechanized Cut-tolength Harvesting System. Silva Fennica, Joensuu, v. 40 (2), p. 335 - 363, 2006.

PARRA, M. O.; CAREY, P. B. Consideraciones metodológicas para la evaluación de la cortadoraprocesadora (harvester) en operaciones forestales. Bosque, Valdivia, v. 21, n. 2, p. 137 - 146, 2000.

SILVA, E. N. da.; CARDOSO MACHADO, C.; MINETTE, L. J.; SOUZA, A. P. de.; FERNANDES, H. C.; SILVA, M. L. da.; JACOVINE, L. A. Avaliação técnica e econômica do corte mecanizado de Pinus sp. com harvester. Revista Árvore, Viçosa, v. 34, n. 4, p. 745 - 753, 2010.

SIMÕES, D.; FENNER, P. T.; TSUTSUI ESPERANCINI, M. S. Avaliação técnica e econômica da colheita de florestas de eucalipto com harvester. Scientia Forestalis, Piracicaba, v. 38, n. 88, p. 611 $618,2010$.

SIRÉN, M.; AALTIO, H. Productivity and Costs of Thinning Harvesters and Harvester-Forwarders. International Journal of Forest Engineering, Seattle, v. 14, n. 1, p. 39 - 48, 2003.

STÖHR, G. W. D. Cálculo de custos de máquinas florestais. Floresta, Curitiba, v.8, n. 2, p. 23 - 30, 1977. 
